\title{
Synthesis of supramolecular receptors for amino acid recognition
}

\author{
Juhi Upadhyaya and Hitesh Parekh ${ }^{\mathrm{a}^{*}}$
}

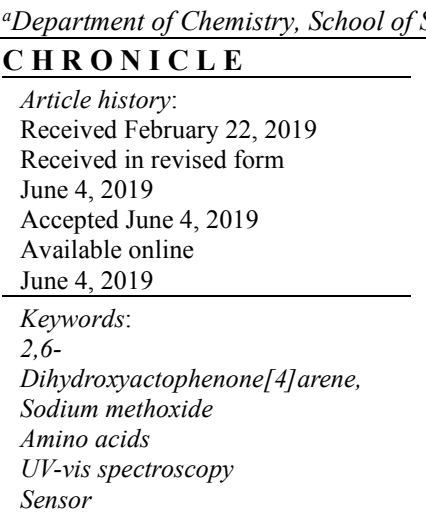

\begin{abstract}
A B S T R A C T
The derivatives of 2,6-dihydroxyacetophenone[4]arene were synthesized via one-step in the presence of base catalyzed cyclocondensation reaction of equimolar volumes of different aliphatic aldehydes and 2,6-dihydroxyacetophenone. All the compounds were purified by column chromatography and characterized by ${ }^{1} \mathrm{H}-\mathrm{NMR}$ Spectroscopy, ${ }^{13} \mathrm{C}-\mathrm{NMR}$ Spectroscopy, IR Spectroscopy, and Mass spectrometry techniques. The compounds were provided with good yield and can be successfully useful for various amino acid detection because of the four ketone groups in 2,6-Dihydroxyaceto-phenone[4]arene were able to react with the amino group of amino acid to give a stable product formation. It was caused by the intermolecular hydrogen bonded between the two groups, ketone and carboxylic group of amino acid. This recognition of amino acid was carried out in UV-vis spectroscopic technique.
\end{abstract}

C 2019 by the authors; licensee Growing Science, Canada.

\section{Introduction}

To understand the study of non-covalent molecular interactions, macrocyclic compounds offer exclusive models. They also create building blocks for molecular and supramolecular designs and producing molecular devices and progressive materials. ${ }^{1}$ Calix[4] arenes are well-known class of bowl like compounds which have received attention due to their simplicity of preparation and tendency to display host-guest complexes. ${ }^{2-3}$ Similarly, it can be carried out in the formation of complexation study ${ }^{4-}$ ${ }^{6}$ and chemical separation. ${ }^{7}$ An effect on the stability of Resorcin[4] arene is the capability to bind toughly with the solvent molecule. ${ }^{8}$ In the resorcinol ring the presence of a vacant ortho position and two hydroxyl groups allows the macrocyclic moleculefor a number of useful synthetic derivatization to form various sophisticated macrocyclic structure. So, Resorcin[4]arenes have been used for the production of various molecular capsules. ${ }^{9}$ The acid catalyzed cyclocondensation reaction of resorcinol, or phenolic compound through some aliphatic aldehydes are well known, since it produces an amorphous mixture of products with high molecular weights. ${ }^{10}$ Furthermore, synthesis of Resorcin[4]arene with base catalyst using formaldehyde was reported. ${ }^{11}$ Different macrocyclic compounds in the Resorcin[4]arenes have customs that Resorcin[4\&6]arenes show different interaction. ${ }^{12}$ Resorcin[4] arene involved in Molecular encapsulation ${ }^{13-14}$ and Metal Organic Framework and different kinds of functionalization. ${ }^{15,16}$ The superior macrocyclic hosts inside the addition of small guest molecules is a growing area of research by a various kind of applications, one of them is recognition of analyte. ${ }^{17-20}$

* Corresponding author. Tel.: +91 79 27541414, Fax: +91 7926301919

E-mail address: keya714@gmail.com (H. Parekh)

(C) 2019 by the authors; licensee Growing Science, Canada

doi: $10.5267 /$ j.ccl.2019.006.002 
The Resorcin[4]arene motifs were prepared by the acid catalyzed condensation reaction between resorcinol and different aldehydes. An electron withdrawing group at the 2-position of resorcinol ring deactivate them for electrophilic substitution reaction, therefore, are very difficult to cyclized with substantial yield and/or form many isomers. The base catalyzed cyclocondensation reaction of 2methylresorcinol with formaldehyde had been reported. ${ }^{11}$ The same research group also reported the reaction of 2-butyrylresorcinol with paraformaldehyde using potassium tert-butoxide as base give a $56 \%$ yield of cyclic tetramer.

In the present efforts, first time we successfully condense 2,6-Dihydroxyacetophenone, with electron withdrawing group at the 2-position, to aliphatic aldehyde. The formerly published articles mostly use paraformaldehyde for condensation but here aliphatic aldehydes successfully used, which can expand the scope of further derivatization. We describe a novel synthesis of new artificial receptors of 2,6-Dihydroxyacetophenone[4]arenes by using $\mathrm{CH}_{3} \mathrm{O}^{-} \mathrm{Na}^{+}$(sodium methoxide) as base. These will provide cyclic structure of the molecule and even arrangement of two hydroxyl groups. ${ }^{21}$ Several artificial receptors were developed for the detection of protein surface. ${ }^{22}$ Disorder of the biotic substances have create very serious difficulties in the human body. Some amino acids like Histidine (His) and cysteine (Cys) deficiency may lead to a metabolic as well as heart problem. Coronary heart disease and Alzheimer's disease and can caused by the absence of said amino acids. ${ }^{23}$ We established a new sensor for detection of biotic amino acid, which is electrophilic due to ketone group to attract the nucleophilic amino acid.

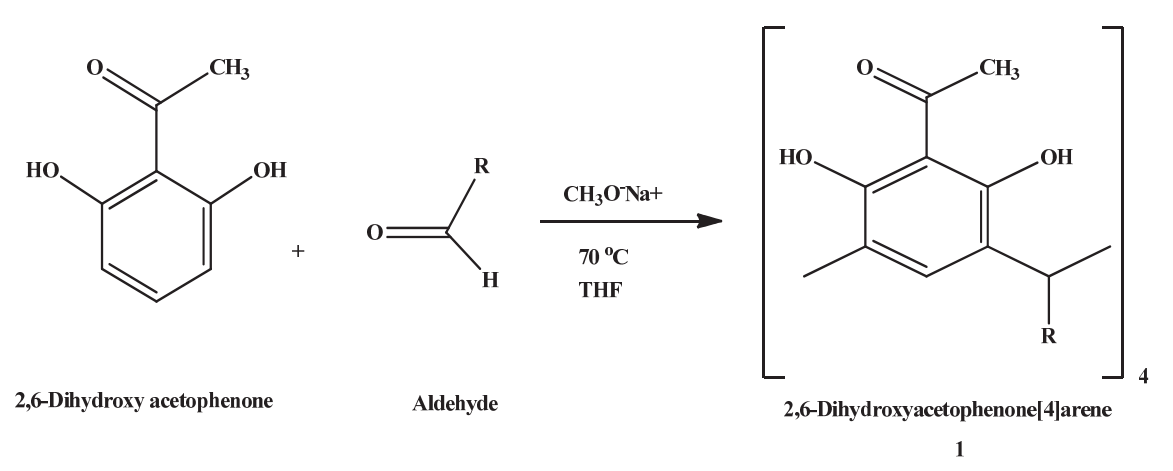

Where, $\mathrm{R}=\mathrm{C}_{2} \mathrm{H}_{5}, \mathrm{C}_{3} \mathrm{H}_{7}, \mathrm{C}_{4} \mathrm{H}_{9}, \mathrm{C}_{5} \mathrm{H}_{11}, \mathrm{C}_{6} \mathrm{H}_{13}, \mathrm{C}_{7} \mathrm{H}_{15}, \mathrm{C}_{8} \mathrm{H}_{17}, \mathrm{C}_{9} \mathrm{H}_{19}$

Scheme: 1 Synthesis of 2,6-dihydroxyacetophenone[4]arene

\subsection{General UV-vis spectra measurement}

The stock solution of sensors 1a-1h was prepared in acetonitrile solvent (sonicated solution). UVvis spectra also recorded in acetonitrile. The stock solutions of amino acids were prepared in distilled water. The UV-vis spectra were display the band of sensors 1a-1h is about around $350 \mathrm{~nm}$. After the addition of amino acid solution, the band shifts at $390 \mathrm{~nm}$.

\section{Result and Discussion}

\subsection{Synthesis}

A condensation reaction of the 2,6-dihydroxyactophenone with aliphatic aldehyde using strong base sodium methoxide in solvent THF at reflux temperature have been successfully carried out to get a good product and a stable structural formation of 2,6-dihydroxyacetophenone[4]arene. All the synthesized compounds have tetrameric bowl shape ring formation. In this synthesis we modified the synthetic pathway that is reported earlier for the condensation reaction between 2-butyrylresorcinol and paraformaldehyde in the presence of potassium tert-butoxide. ${ }^{11}$ We tried this procedure for 2,6dihydroxyacetophenone with aromatic aldehyde but it was not proceed at al. We developed a new 
synthetic pathway for 2,6-Dihydroxyacetophenone[4]arene synthesis. For the synthesis of target molecule, we had tried various acids like, Conc. $\mathrm{HCl}$ and PTSA (para-toluene sulfonic acid). Furthermore, we also tried different bases like TEA (triethylamine), $\mathrm{C}_{5} \mathrm{H}_{5} \mathrm{~N}$ (pyridine), $\mathrm{NaOH}$ (sodium hydroxide), and $\mathrm{KOH}$ (potassium hydroxide). However, the reaction was not complete. Finally, $\mathrm{CH}_{3} \mathrm{O}^{-}$ $\mathrm{Na}^{+}$used as a base for completion of reaction with high yield. $\mathrm{CH}_{3} \mathrm{O}^{-} \mathrm{Na}^{+}$is strong base and methoxide pulls proton to give methanol so it can be used in many organic synthesis. Methoxide pulls proton, hence it is effectively working in the completion of this reaction with good yield and lower isomer formation, than the other bases. The long chain aliphatic aldehydes were condensed effectively than aromatic aldehyde because of the steric hindrance of the aromatic ring.

${ }^{1} \mathrm{H}$ and ${ }^{13} \mathrm{C}$-NMR spectra were recorded using $400 \mathrm{MHz}$ Bruker Avance spectrometer. ${ }^{1} \mathrm{H}-\mathrm{NMR}$ carried out in DMSO- $\mathrm{d}_{6}$ and $\mathrm{CDCl}_{3}$. The $\delta$ value of 8 hydroxyl group is shifts around 9 ppm for $\mathbf{1 a}, \mathbf{1 b}$ and $1 \mathbf{c}\left(\mathrm{CDCl}_{3}\right)$, and for another compounds it is observe at around $11 \mathrm{ppm}$ (DMSO- $\left.\mathrm{d}_{6}\right)$, because of the ketonic environment in the 2,6-dihydroxyacetophenone moiety. The characteristic peak of bridge proton found in between 4 to $4.5 \mathrm{ppm}$ as triplet, it shows that the 2,6-dihydroxyactophenone[4] arene formed because of the absence of peaks of free aldehyde. Aldehyde is condensed at $3^{\text {rd }}$ and $5^{\text {th }}$ position of two separate 2,6-Dihydroxyacetophenone to formed the bridge between two parent ring and formed cyclic structure. The proton of 2,6-Dihydroxyacetophenone ring is observe at around $7.2 \mathrm{ppm}$. The peaks at around $1.20 \mathrm{ppm}$ and $0.80 \mathrm{ppm}$ show proton of respective aliphatic chain. IR spectra were performed in the range of $400 \mathrm{~cm}^{-1}$ to $4000 \mathrm{~cm}^{-1}$. The IR spectrum shows a broad absorption peak of hydroxyl group in between $3100-3400 \mathrm{~cm}^{-1}$. The sharp bend of $\mathrm{C}=\mathrm{O}$ stretching was shows at 1622.57 $\mathrm{cm}^{-1}$. The aldehyde- $\mathrm{CH}_{2}$ - chain shows absorbance peak at around $1447.17 \mathrm{~cm}^{-1}$ in the spectrum. ESIMS was performed on a XEVO-G2SQTOF, Chloroform had been used as mobile phase, electron spray ionization (ESI) is used as ion source in both the channels $(-\mathrm{Ve})$ as well as $(+\mathrm{Ve})$. The obtained result showed molecular ion peaks at $\mathrm{m} / \mathrm{z}$ 769.32, 825.39, 881.45, 937.30, 993.29, 1049.40, 1105.50, 1161.61, (Figs. 15-22) in good agreement with the molecular formulas of 1a, 1b, 1c,1d, 1e, 1f, 1g, 1h, respectively. Physical properties of all compounds are listed in table 1.

\subsection{Detection of amino acid}

There are significant role of Amino acid decarboxylases in numerous diverse biological pathways in both plants and animals, like, smooth muscle contraction, gastric acid secretion, immune regulation, allergic response, and wound healing in animals as well as cell explosion and distinction, invisibility and growth, flower induction and development, embryogenesis, fruit-set and growth, and fruit ripening in plants. ${ }^{17,24}$ The substituted calix[4]arene over by carboxylic groups shows the interaction with arginine, and lysine also reported. ${ }^{25}$ The different types of amines had been used for the synthesis and observe receptor properties of heterofunctionalized derivative of $r c t t$ - $C$-naphthyl resorcinarene. ${ }^{26}$ The main aim of our work is to develop a sensor molecule which can recognize amines in aqueous solution. The interaction of sensors with the amino acid receptor have done by the electrophilic nature of ketonic group in the 2,6-dihydroxyacetophenone[4] arene ring, which will attract to nucleophilic group of amino acid. The 2,6-dihydroxyacetophenone[4]arene having four ketone groups that is reserved to react with the amino group of amino acid. Here, the detection of amino acids has been carried out by UV-vis spectroscopic technique. For the detection of amino acid, the concentration of sensor and amino acid were taken about $1 \times 10^{-5} \mathrm{M}$ in acetonitrile solvent and $1 \times 10^{-4} \mathrm{M}$ in distilled water respectively. The observation of amino acid detection was done by adding amino acid progressively into the sensor solution. The UV-vis spectroscopy experiments of all the sensors have observed the $\lambda_{\max }$ value at around $350 \mathrm{~nm}$. After addition of amino acid it has shown increasing at around $390 \mathrm{~nm}$ because of new compound formation. The isobestic point is at $370 \mathrm{~nm}$ which indicates that the new product formation have done after the addition of amino acid. Here, we tested three different amino acids which are nucleophilic in nature. Ketonic group of sensor and amino acid group of amine compounds react together and it will produce intermolecular hydrogen bond between two groups, which will give stable structural formation of the final product after the titration. We also tried amino acid with 2,6- 
Dihydroxyactophenone (Monomer) but it shows very less binding with amino acid then synthesized supramolecular ring. In 2,6-Dihydroxyacetophenone[4] arene having tetramer ring gives more effective detection for amino acid.

\subsubsection{UV-vis spectra of detecting Glycine}

Glycine solution was prepared in distilled water and sensor 1a-1h solutions were prepared in acetonitrile solvent. The Figs. (28-35) displayed the changes in UV-vis spectra after the addition of glycine. In the UV-vis spectra, sensor $1 \mathbf{a}-1 \mathbf{h}\left(1 \times 10^{-5} \mathrm{M}\right)$ shows the absorption peak at around $350 \mathrm{~nm}$. However, after the addition by different volumes of glycine solution $\left(1 \times 10^{-4} \mathrm{M}\right)$ the new absorption peak observed at around $390 \mathrm{~nm}$. In the UV-vis spectra, the isobestic point is about $370 \mathrm{~nm}$. It is indicating that the new compound is formed after the addition of Glycine.

\subsection{2. $U V$-vis spectra of detecting L-phenylalanine (LPA)}

A solution of L-Phenylalanine was prepared in distilled water and sensor solutions $\mathbf{1 a - 1 h}$ were prepared in acetonitrile solvent. The figures 36 to 43 display the changes in UV-vis spectra after the addition of L-phenylalanine. In the UV-vis spectra, sensor $1 \mathbf{a}-1 \mathbf{h}\left(1 \times 10^{-5} \mathrm{M}\right)$ displayed the absorption peak at around $350 \mathrm{~nm}$. But, after the addition with different volumes of L-phenylalanine solution $\left(1 \times 10^{-4} \mathrm{M}\right)$ the new absorption peak appeared at around $390 \mathrm{~nm}$. The isobestic point is around at 370 $\mathrm{nm}$, is indicates that the new compound is formed after the titration of L-Phenylalanine.

\subsubsection{UV-vis spectra of detecting L-phenylglycine ( $L P G)$}

In the same way L-phenylglycine solution was prepared in distilled water and solutions of sensor 1a-1h were prepared in acetonitrile solvent. The changes in UV-vis spectra after the addition of Lphenylglycine have been shown in the Figs. (44-51). Sensor $1 \mathrm{a}-1 \mathrm{~h}\left(1 \times 10^{-5} \mathrm{M}\right)$ shows the absorption peak at around $350 \mathrm{~nm}$. After the titration using different volumes of L-phenylglycine solution $\left(1 \times 10^{-}\right.$ ${ }^{4} \mathrm{M}$ ), the new absorption peak obtained at around $390 \mathrm{~nm}$. The formation of new compound after the addition of L-phenylglycine was confirmed by the isobestic point at around $370 \mathrm{~nm}$.

\section{Conclusion}

In conclusion, we have successfully synthesized 2,6-Dihydroxyacetophenone[4]arene derivatives by using $\mathrm{CH}_{3} \mathrm{O}^{-} \mathrm{Na}^{+}$as base under the reflux condition. All the prepared compounds were characterized by ${ }^{1} \mathrm{H}-\mathrm{NMR},{ }^{13} \mathrm{C}-\mathrm{NMR}$, IR, and Mass spectrometry techniques. Prepared compounds successfully employed as sensor for the different types of amino acid. Here, all the compounds are working as sensor for the recognition of glycine, L-phenyl alanine, L-phenylglycine. Amino acid interacts with the four ketone group of sensor as well as four hydroxyl groups to form a Hydrogen bond. The interactions of sensors with different amino acids have shown by UV-vis spectroscopy. Amino acids are able to interact with sensors due to the electrophilic nature of sensor. Hydrogen bond was taken advantage to stabilize the product of titration. The interactions involve in the detection of amino acid is hydrogen bonding interaction (Fig. 52). The observation shows that tetrameric supramolecular ring is more effective for amino acid detection compare with the 2,6-Dihydroxyacetophenone.

\section{Acknowledgements}

We are thankful to the Head of Chemistry Department, Gujarat University for necessary laboratory facilities and UGC-BSR for Research start up grant for financial support.

\section{Declaration of interest}

The authors report no declaration of interest. 


\section{Experimental}

\subsection{Instruments, Reagents and Methods}

All solvents and reagents were used of L.R grade without further purification, and the aldehydes were purchased from Sigma Aldrich. TLC was run on Aluminum pre-coated ready-made thin layer chromatographic (TLC) silica gel 60 F254 plate (Merck, Germany) and visualization was done using iodine or UV light. Melting points were verified by programmable Veego melting point apparatus. ${ }^{1} \mathrm{H}-$ NMR (DMSO-d6/CDCl 3 ) and ${ }^{13} \mathrm{C}$-NMR $\left(\mathrm{CDCl}_{3}\right)$ were recorded through Bruker Avance $(400 \mathrm{MHz})$ spectrometer. IR spectral data were recorded using Perkin Elmer FT-IR 377 spectrometer using $\mathrm{KBr}$ powder as standard. Mass spectra were carried out by XEVO-G2SQTOF. Elemental analysis was recorded in EURO VECTOR EA3000 CHNS-O Analyzer. Absorption spectra were measured in Jasco V-630 UV-vis spectrophotometer.

4.2. Synthesis of 4,6,10,12,16,18,22,24-octahydroxy-5,11,17,23-tetraacetyl-2,8,14,20tetraethylresorcin [4]arene (1a)

2,6-dihydroxyacetophenone $(0.500 \mathrm{gm}, 3.289 \mathrm{mmol})$ with propanal $(0.23 \mathrm{~mL}, 3.289 \mathrm{mmol})$ was taken in $20 \mathrm{ml}$ THF and during stirring add $\mathrm{CH}_{3} \mathrm{O}^{-} \mathrm{Na}^{+}(0.1 \mathrm{gm}, 1.85 \mathrm{mmol})$ at room temperature. The Reaction mixture was then heated at reflux condition with constant stirring for 72 hours. The completion of reaction was checked by TLC (Hexane: Ethyl acetate, 8:2). After completion of reaction, the reaction mixture had been cooled at room temperature and solvent was evaporated in vacuum. The crude product was dissolve in $25 \mathrm{ml}$ of Methanol. A few drops of $\mathrm{AcOH}$ (acetic acid) was added in this solution to get $7 \mathrm{pH}$. The yellow precipitate was formed and stirred at $0-5{ }^{\circ} \mathrm{C}$ for $30 \mathrm{mins}$. The product was then filtrated and washed with cold methanol and purified by column chromatography using ethyl acetate and hexane and dried at $40-45{ }^{\circ} \mathrm{C}$ temperature. ${ }^{1} \mathrm{H}-\mathrm{NMR}\left(\mathrm{CDCl}_{3}\right), \delta$, ppm: $9.02 \mathrm{~s}(8 \mathrm{H}, \mathrm{Ar}-\mathrm{OH})$, $7.39 \mathrm{~s}(4 \mathrm{H}, \mathrm{Ar}), 4.29 \mathrm{t}(4 \mathrm{H}, J=7.6 \mathrm{MHz},-\mathrm{CH}-), 2.72 \mathrm{~s}\left(12 \mathrm{H},-\mathrm{CH}_{3} \mathrm{CO}\right), 2.23 \mathrm{q}\left(8 \mathrm{H}, J=16 \mathrm{MHz},-\mathrm{CH}_{2}-\right)$, $0.94 \mathrm{t}\left(12 \mathrm{H}, J=7.2 \mathrm{MHz},-\mathrm{CH}_{3}\right)$. MS $m / z:=769.32[\mathrm{M}]^{+}, 770.33[\mathrm{M}+\mathrm{H}]^{+}$. Anal. Calcd. for $\mathrm{C}_{44} \mathrm{H}_{48} \mathrm{O}_{12}(\%)$ : C, 68.74; H, 6.29; O, 24.97. Found: C, 68.71; H, 6.26; O, 24.96.

The compounds $\mathbf{1 b}-\mathbf{1 h}$ have been synthesized by using a similar pathway by changing respective aldehydes like Butanal $(0.29 \mathrm{~mL}, 3.289 \mathrm{mmol})$, pentanal $(0.35 \mathrm{~mL}, 3.289 \mathrm{mmol})$, Hexanal $(0.39 \mathrm{~mL}$, $3.289 \mathrm{mmol})$ Heptanal $(0.46 \mathrm{~mL}, 3.289 \mathrm{mmol})$, Octanal $(0.51 \mathrm{~mL}, 3.289 \mathrm{mmol})$, Nonanal $(0.56 \mathrm{~mL}$, $3.289 \mathrm{mmol})$, and Decanal $(0.60 \mathrm{~mL}, 3.289 \mathrm{mmol})$.

4.3. Synthesis of 4,6,10,12,16,18,22,24-octahydroxy-5,11,17,23-tetraacetyl-2,8,14,20tetrapropylresorcin [4]arene (1b)

${ }^{1} \mathrm{H}-\mathrm{NMR}\left(\mathrm{CDCl}_{3}\right) \delta$, ppm: $9.02 \mathrm{~s}(8 \mathrm{H}, \mathrm{Ar}-\mathrm{OH}), 7.40 \mathrm{~s}(4 \mathrm{H}, \mathrm{Ar}), 4.41 \mathrm{t}(4 \mathrm{H}, J=7.6 \mathrm{MHz},-\mathrm{CH}-), 2.72 \mathrm{~s}$ $\left(12 \mathrm{H},-\mathrm{CH}_{3} \mathrm{CO}\right), 2.16 \mathrm{q}\left(8 \mathrm{H}, J=7.2 \mathrm{MHz},-\mathrm{CH}_{2}-\right), 1.34 \mathrm{~m}\left(8 \mathrm{H}, J=14 \mathrm{MHz},-\mathrm{CH}_{2}-\mathrm{CH}_{2}-\right), 0.98 \mathrm{t}$ $\left(12 \mathrm{H}, J=14.4 \mathrm{MHz},-\mathrm{CH}_{3}\right) . \mathrm{MS} m / z:=825.39[\mathrm{M}]^{+}, 826.39[\mathrm{M}+\mathrm{H}]^{+}$. Anal. Calcd. for $\mathrm{C}_{48} \mathrm{H}_{56} \mathrm{O}_{12}(\%): \mathrm{C}$, 69.88; H, 6.84; O, 23.27. Found: C, 69.85; H, 6.82; O, 23.25.

4.4. Synthesis of 4,6,10,12,16,18,22,24-octahydroxy-5,11,17,23-tetraacetyl-2,8,14,20tetrabutylresorcin [4]arene (1c)

${ }^{1} \mathrm{H}-\mathrm{NMR}\left(\mathrm{CDCl}_{3}\right) \delta$, ppm: $9.04 \mathrm{~s}(8 \mathrm{H}, \mathrm{Ar}-\mathrm{OH}), 7.43 \mathrm{~s}(4 \mathrm{H}, \mathrm{Ar}), 4.41 \mathrm{t}(4 \mathrm{H}, J=7.6 \mathrm{MHz},-\mathrm{CH}-), 2.72 \mathrm{~s}$ $\left(12 \mathrm{H},-\mathrm{CH}_{3} \mathrm{CO}\right), 2.21 \mathrm{q}\left(8 \mathrm{H}, J=7.6 \mathrm{MHz},-\mathrm{CH}_{2}-\right), 1.48 \mathrm{~m}\left(16 \mathrm{H}, J=29.2 \mathrm{MHz},-\mathrm{CH}_{2}-\mathrm{CH}_{2}-\right), 0.97 \mathrm{t}(12 \mathrm{H}$, $\left.J=14.4 \mathrm{MHz},-\mathrm{CH}_{3}\right)$. MS $m / z:=881.45[\mathrm{M}+\mathrm{H}]^{+}$. Anal. Calcd. for $\mathrm{C}_{52} \mathrm{H}_{64} \mathrm{O}_{12}(\%): \mathrm{C}, 70.89 ; \mathrm{H}, 7.32 ; \mathrm{O}$, 21.79. Found: C, 70.85; H, 7.30; O, 21.75.

4.5. Synthesis of 4,6,10,12,16,18,22,24-octahydroxy-5,11,17,23-tetraacetyl-2,8,14,20tetrapentylresorcin [4] arene (1d)

${ }^{1} \mathrm{H}-\mathrm{NMR}(\mathrm{DMSO}) \delta$, ppm: $11.33 \mathrm{~s}(8 \mathrm{H}, \mathrm{Ar}-\mathrm{OH}), 7.46 \mathrm{~s}(4 \mathrm{H}, \mathrm{Ar}), 4.42 \mathrm{t}(4 \mathrm{H}, J=7.6 \mathrm{MHz}, \mathrm{CH}-), 2.59 \mathrm{~s}$ $\left(12 \mathrm{H},-\mathrm{CH}_{3} \mathrm{CO}\right), 2.06 \mathrm{q}\left(8 \mathrm{H}, J=6.4 \mathrm{MHz},-\mathrm{CH}_{2}-\right), 1.25 \mathrm{~m}\left(24 \mathrm{H}, J=14.8 \mathrm{MHz},-\mathrm{CH}_{2}-\mathrm{CH}_{2}-\right), 0.85 \mathrm{t}(12 \mathrm{H}$, $\left.J=14 \mathrm{MHz},-\mathrm{CH}_{3}\right) .{ }^{13} \mathrm{C}-\mathrm{NMR}\left(\mathrm{CDCl}_{3}\right), \delta \mathrm{c}, \mathrm{ppm}:=14.12,22.70,27.64,31.87,32.55,33.57,33.88$, $110.63,123.32,123.66,129.96,155.57,157.08,207.29 . \mathrm{MS} m / z:=937.30,[\mathrm{M}]^{+}, 938.31,[\mathrm{M}+\mathrm{H}]^{+}$. Anal. Calcd. for $\mathrm{C}_{56} \mathrm{H}_{72} \mathrm{O}_{12}(\%)$ : C, 71.77; H, 7.74; O, 20.49. Found: C, 71.74; H, 7.73; O, 20.48. IR,v, $\mathrm{cm}^{-1}$ : 3279.92 (O-H str.), $1622.57(\mathrm{C}=\mathrm{O}), 1447.86\left(-\mathrm{CH}_{2}-\right), 1369.52\left(-\mathrm{CH}_{3}\right), 905.91(=\mathrm{C}-\mathrm{H})$. 
4.6. Synthesis of 4,6,10,12,16,18,22,24-octahydroxy-5,11,17,23-tetraacetyl-2,8,14,20tetrahexylresorcin [4] arene (1e)

${ }^{1} \mathrm{H}-\mathrm{NMR}$ (DMSO) $\delta$, ppm: $11.58 \mathrm{~s}(8 \mathrm{H}, \mathrm{Ar}-\mathrm{OH}), 7.46 \mathrm{~s}(4 \mathrm{H}, \mathrm{Ar}), 4.40 \mathrm{t}(4 \mathrm{H}, J=8 \mathrm{MHz},-\mathrm{CH}-), 2.59 \mathrm{~s}$ $\left(12 \mathrm{H},-\mathrm{CH}_{3} \mathrm{CO}\right), 2.07 \mathrm{q}\left(8 \mathrm{H}, J=6.4 \mathrm{MHz},-\mathrm{CH}_{2}-\right), 1.21 \mathrm{~m}\left(32 \mathrm{H}, J=10.4 \mathrm{MHz},-\mathrm{CH}_{2}-\mathrm{CH}_{2}-\right), 0.85 \mathrm{t}(12 \mathrm{H}$, $\left.J=13.6 \mathrm{MHz},-\mathrm{CH}_{3}\right) .{ }^{13} \mathrm{C}-\mathrm{NMR}\left(\mathrm{CDCl}_{3}\right), \delta \mathrm{c}, \mathrm{ppm}:=14.06,22.65,27.90,29.32,31.87,32.51,33.61$, $33.88,110.63,123.31,123.64,129.98,155.56,157.08,207.28$. MS $m / z:=993.29[\mathrm{M}]^{+}$, 994.29, $[\mathrm{M}+\mathrm{H}]^{+}$. Anal. Calcd. for $\mathrm{C}_{60} \mathrm{H}_{80} \mathrm{O}_{12}(\%)$ : C, 72.55; H, 8.12; O, 19.33. Found: C, 72.51; H, 8.10; O, 19.31. IR, v, cm ${ }^{-1}: 3290.70$ (O-H str.), $1617.71(\mathrm{C}=\mathrm{O}), 1447.17\left(-\mathrm{CH}_{2}-\right), 1366.49\left(-\mathrm{CH}_{3}\right), 918.37$ (=C-H).

4.7. Synthesis of 4,6,10,12,16,18,22,24-octahydroxy-5,11,17,23-tetraacetyl2,8,14,20tetraheptylresorcin [4]arene (1f)

${ }^{1} \mathrm{H}-\mathrm{NMR}$ (DMSO) $\delta$, ppm: $11.39 \mathrm{~s}(8 \mathrm{H}, \mathrm{Ar}-\mathrm{OH}), 7.44 \mathrm{~s}(4 \mathrm{H}, \mathrm{Ar}), 4.42 \mathrm{t}$ (4H, J=7.6 MHz,-CH-), $2.59 \mathrm{~s}$ $\left(12 \mathrm{H},-\mathrm{CH}_{3} \mathrm{CO}\right), 2.05 \mathrm{q}\left(8 \mathrm{H}, J=6.4 \mathrm{MHz},-\mathrm{CH}_{2}-\right), 1.24 \mathrm{~m}\left(40 \mathrm{H}, J=14.4 \mathrm{MHz},-\mathrm{CH}_{2}-\mathrm{CH}_{2}-\right), 0.84 \mathrm{t}(12 \mathrm{H}$, $\left.J=13.2 \mathrm{MHz},-\mathrm{CH}_{3}\right) .{ }^{13} \mathrm{C}-\mathrm{NMR}\left(\mathrm{CDCl}_{3}\right), \delta \mathrm{c}, \mathrm{ppm}:=14.10,22.64,27.92,29.35,29.58,31.84,32.48$, $33.59,33.87,110.62,123.30,123.63,129.98,155.76,157.08,207.28 . \mathrm{MS} m / z:=1049.40[\mathrm{M}]^{+}$, 1050.30, $[\mathrm{M}+\mathrm{H}]^{+}$. Anal. Calcd. for $\mathrm{C}_{64} \mathrm{H}_{88} \mathrm{O}_{12}(\%)$ : C, 73.25; H, 8.45; O, 18.30. Found: C, 73.22; H, 8.44; O, 18.28. IR, v, cm ${ }^{-1}: 3339.97$ (O-H str.), $1613.54(\mathrm{C}=\mathrm{O}), 1447.71\left(-\mathrm{CH}_{2}-\right), 1358.88\left(-\mathrm{CH}_{3}\right)$, $902.27(=\mathrm{C}-\mathrm{H})$.

4.8. Synthesis of 4,6,10,12,16,18,22,24-octahydroxy-5,11,17,23-tetraacetyl-2,8,14,20tetraoctylresorcin [4] arene (1g)

${ }^{1} \mathrm{H}-\mathrm{NMR}(\mathrm{DMSO}) \delta \mathrm{ppm}: 11.18 \mathrm{~s}(8 \mathrm{H}, \mathrm{Ar}-\mathrm{OH}), 7.41 \mathrm{~s}(4 \mathrm{H}, \mathrm{Ar}), 4.43 \mathrm{t}(4 \mathrm{H}, J=7.2 \mathrm{MHz}, \mathrm{CH}-), 2.66$ (s, $\left.12 \mathrm{H},-\mathrm{CH}_{3} \mathrm{CO}\right), 2.05 \mathrm{q}\left(8 \mathrm{H}, J=5.2 \mathrm{MHz},-\mathrm{CH}_{2}-\right), 1.20 \mathrm{~m}\left(48 \mathrm{H}, J=36.4 \mathrm{MHz},-\mathrm{CH}_{2}-\mathrm{CH}_{2}-\right), 0.80 \mathrm{t}(12 \mathrm{H}$, $\left.J=12.8 \mathrm{MHz},-\mathrm{CH}_{3}\right) .{ }^{13} \mathrm{C}-\mathrm{NMR}\left(\mathrm{CDCl}_{3}\right), \delta \mathrm{c}, \mathrm{ppm}:=14.14,22.71,27.93,29.34,29.64,29.67,31.87$, $32.49,33.60,33.88,110.63,123.30,123.64,130.00,155.77,157.09,207.28 . \mathrm{MS} m / z:=1105.50[\mathrm{M}]$ ${ }^{+}$. Anal. Calcd. for $\mathrm{C}_{68} \mathrm{H}_{96} \mathrm{O}_{12}(\%)$ : C, 73.88; H, 8.75; O, 17.37. Found: C, 73.86; H, 8.75; O, 17.35.IR, $v, \mathrm{~cm}^{-1}: 3305.65(\mathrm{O}-\mathrm{H}$ str. $), 1617.82(\mathrm{C}=\mathrm{O}), 1447.32\left(-\mathrm{CH}_{2}-\right), 1368.33\left(-\mathrm{CH}_{3}\right), 887.17(=\mathrm{C}-\mathrm{H})$.

4.9. Synthesis of 4,6,10,12,16,18,22,24-octahydroxy-5,11,17,23-tetraacetyl-2,8,14,20tetranonylresorcin [4] arene (1h)

${ }^{1} \mathrm{H}-\mathrm{NMR}$ (DMSO) $\delta$, ppm: $11.66 \mathrm{~s}(8 \mathrm{H}, \mathrm{Ar}-\mathrm{OH}), 7.42 \mathrm{~s}(4 \mathrm{H}, \mathrm{Ar}), 4.40 \mathrm{t}(4 \mathrm{H}, J=7.3 \mathrm{MHz}$, -CH-), 2.66 $\left(\mathrm{s}, 12 \mathrm{H},-\mathrm{CH}_{3} \mathrm{CO}\right), 2.06 \mathrm{q}\left(8 \mathrm{H}, J=5.2 \mathrm{MHz},-\mathrm{CH}_{2}-\right), 1.20 \mathrm{~m}\left(56 \mathrm{H}, J=36.8 \mathrm{MHz},-\mathrm{CH}_{2}-\mathrm{CH}_{2}-\right), 0.81 \mathrm{t}(12 \mathrm{H}$, $\left.J=7.2 \mathrm{MHz},-\mathrm{CH}_{3}\right) .{ }^{13} \mathrm{C}-\mathrm{NMR}\left(\mathrm{CDCl}_{3}\right), \delta \mathrm{c}, \mathrm{ppm}:=14.16,22.56,22.74,27.96,29.36,29.66,29.75$, $31.97,32.49,32.70,33.61,33.88,110.64,123.31,123.64,130.00,155.79,157.10,207.27 . \mathrm{MS} \mathrm{m} / \mathrm{z}:=$ $1161.61[\mathrm{M}]^{+}, 1162.62,[\mathrm{M}+\mathrm{H}]^{+}$. Anal. Calcd. for $\mathrm{C}_{72} \mathrm{H}_{104} \mathrm{O}_{12}(\%)$ : C, 74.45; H, 9.02; O, 16.53. Found: C, 74.44; H, 9.00; O, 16.51.IR, v, cm ${ }^{-1}: 3273.00$ (O-H str.), 1620.90 (C=O), $1448.89\left(-\mathrm{CH}_{2-}\right), 1370.36$ $\left(-\mathrm{CH}_{3}\right), 905.34(=\mathrm{C}-\mathrm{H})$.

Table. 1. Physical properties of synthesized compounds

\begin{tabular}{cccccc}
\hline $\begin{array}{c}\text { Compound } \\
\text { Name }\end{array}$ & $\begin{array}{c}\text { Chemical } \\
\text { Formula }\end{array}$ & Color & $\begin{array}{c}\text { Molecular } \\
\text { weight }\end{array}$ & $\begin{array}{c}\text { Melting } \\
\text { Point }\end{array}$ & \% of yield \\
\hline $1 \mathrm{a}$ & $\mathrm{C}_{44} \mathrm{H}_{48} \mathrm{O}_{12}$ & Yellow & 769 & $265^{\circ} \mathrm{C}$ & $65 \%$ \\
$1 \mathrm{~b}$ & $\mathrm{C}_{48} \mathrm{H}_{56} \mathrm{O}_{12}$ & Yellow & 825 & $244^{\circ} \mathrm{C}$ & $65 \%$ \\
$1 \mathrm{c}$ & $\mathrm{C}_{52} \mathrm{H}_{64} \mathrm{O}_{12}$ & Yellow & 880 & $251^{\circ} \mathrm{C}$ & $70 \%$ \\
$1 \mathrm{~d}$ & $\mathrm{C}_{56} \mathrm{H}_{72} \mathrm{O}_{12}$ & Yellow & 937 & $226^{\circ} \mathrm{C}$ & $70 \%$ \\
$1 \mathrm{e}$ & $\mathrm{C}_{60} \mathrm{H}_{80} \mathrm{O}_{12}$ & Yellow & 993 & $150^{\circ} \mathrm{C}$ & $70 \%$ \\
$1 \mathrm{f}$ & $\mathrm{C}_{64} \mathrm{H}_{88} \mathrm{O}_{12}$ & Yellow & 1049 & $116^{\circ} \mathrm{C}$ & $85 \%$ \\
$1 \mathrm{~g}$ & $\mathrm{C}_{68} \mathrm{H}_{96} \mathrm{O}_{12}$ & Yellow & 1105 & $124^{\circ} \mathrm{C}$ & $60 \%$ \\
$1 \mathrm{~h}$ & $\mathrm{C}_{72} \mathrm{H}_{104} \mathrm{O}_{12}$ & Yellow & 1162 & $112^{\circ} \mathrm{C}$ & $60 \%$ \\
\hline
\end{tabular}




\section{Figures}

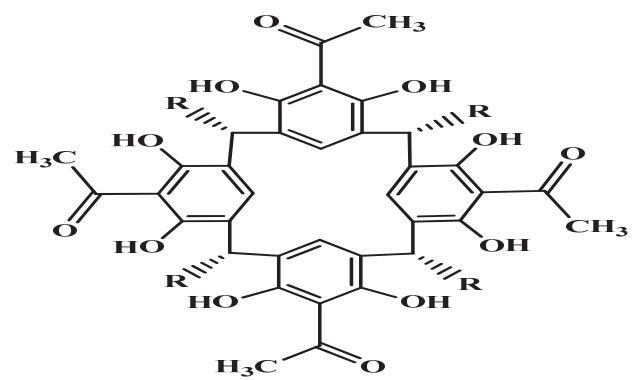

Fig. 1. Structure of 2,6-dihydroxyacetophenone[4]arene

where, $\mathrm{R}=\mathrm{C}_{2} \mathrm{H}_{5}(\mathbf{1 a}), \mathrm{R}=\mathrm{C}_{3} \mathrm{H}_{7}(\mathbf{1 b}), \mathrm{R}=\mathrm{C}_{4} \mathrm{H}_{9}(\mathbf{1} \mathbf{c}), \mathrm{R}=\mathrm{C}_{5} \mathrm{H}_{11}(\mathbf{1 d}), \mathrm{R}=\mathrm{C}_{6} \mathrm{H}_{13}(\mathbf{1 e}), \mathrm{C}_{7} \mathrm{H}_{15}(\mathbf{1 f}), \mathrm{R}=\mathrm{C}_{8} \mathrm{H}_{17}(\mathbf{1 g}), \mathrm{R}=\mathrm{C}_{9} \mathrm{H}_{19}$

(1h)

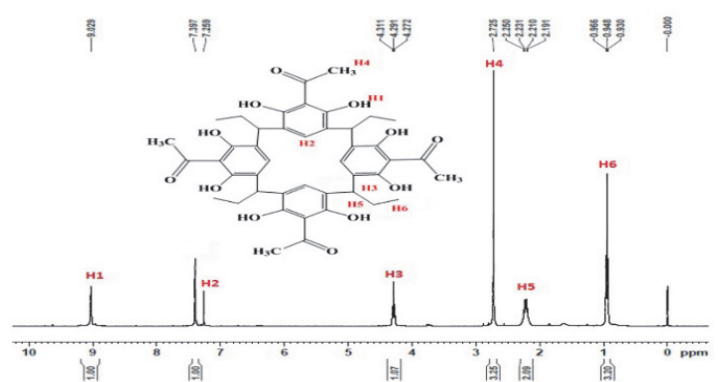

Fig. 2. ${ }^{1} \mathrm{H}-\mathrm{NMR}$ spectrum of compound $1 \mathrm{a}$

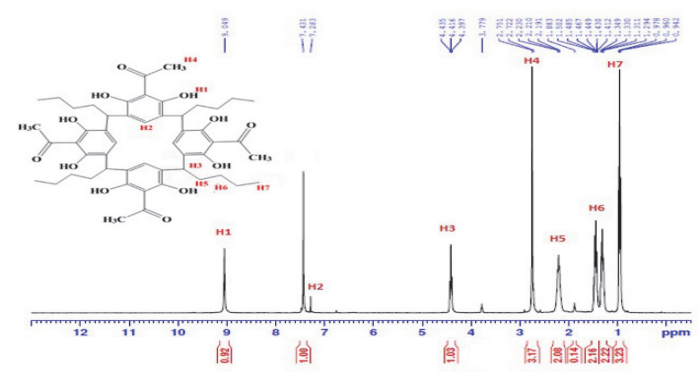

Fig. 4. ${ }^{1} \mathrm{H}-\mathrm{NMR}$ spectrum of compound $1 \mathrm{c}$

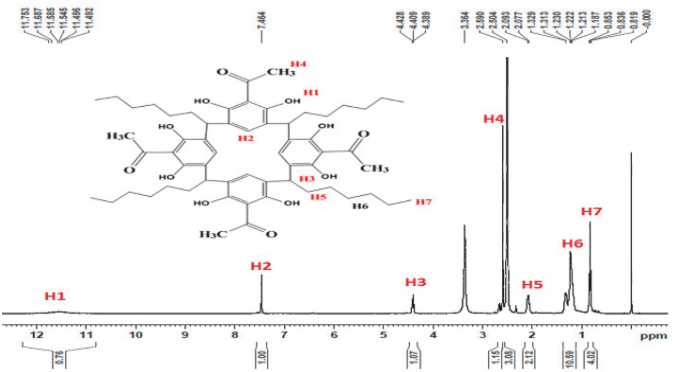

Fig. 6. ${ }^{1} \mathrm{H}-\mathrm{NMR}$ spectrum of compound $1 \mathrm{e}$

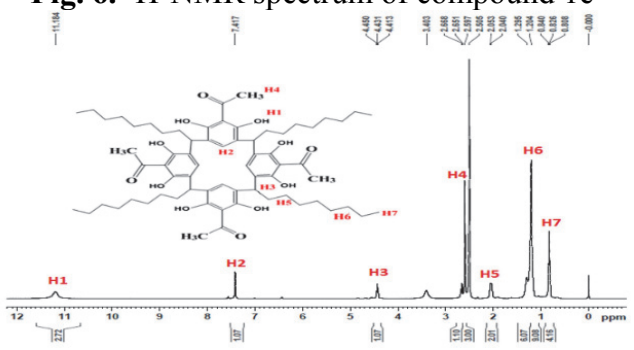

Fig. 8. ${ }^{1} \mathrm{H}-\mathrm{NMR}$ spectrum of compound $1 \mathrm{~g}$

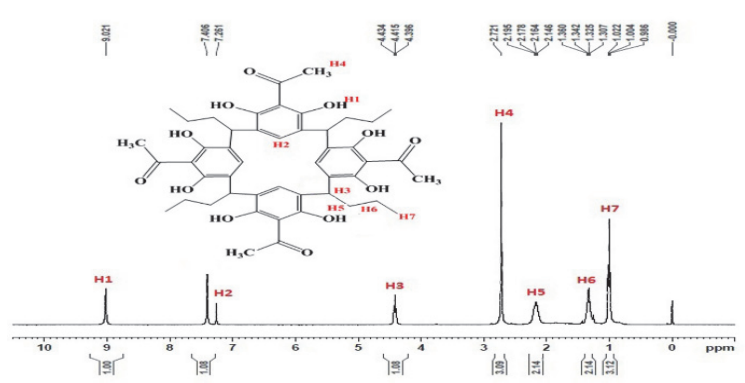

Fig. 3. ${ }^{1} \mathrm{H}-\mathrm{NMR}$ spectrum of compound $1 \mathrm{~b}$

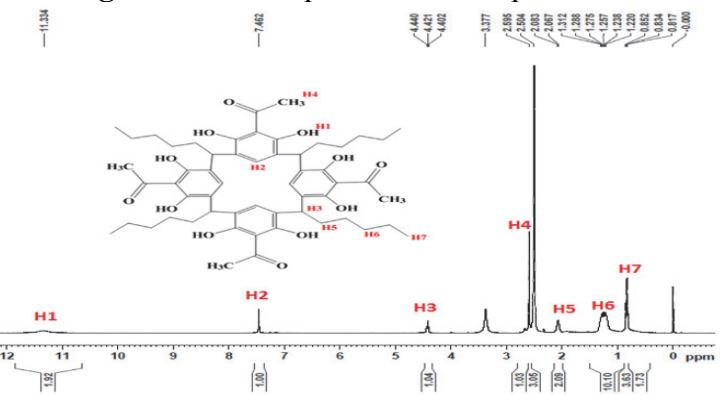

Fig. 5. ${ }^{1} \mathrm{H}-\mathrm{NMR}$ spectrum of compound $1 \mathrm{~d}$

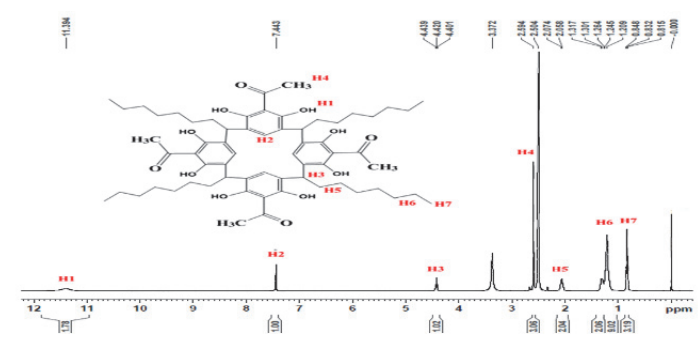

Fig. 7. ${ }^{1} \mathrm{H}-\mathrm{NMR}$ spectrum of compound if

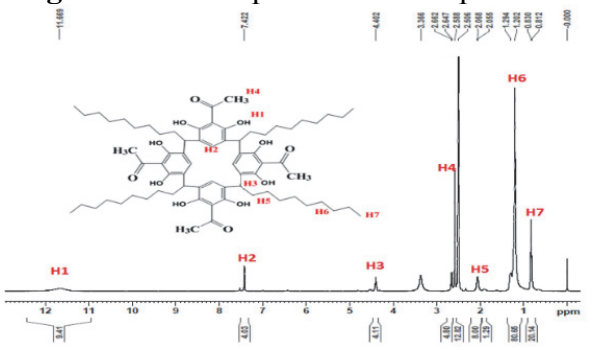

Fig. 9. ${ }^{1} \mathrm{H}-\mathrm{NMR}$ spectrum of compound $1 \mathrm{~h}$ 


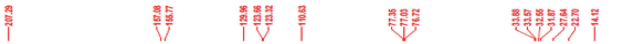
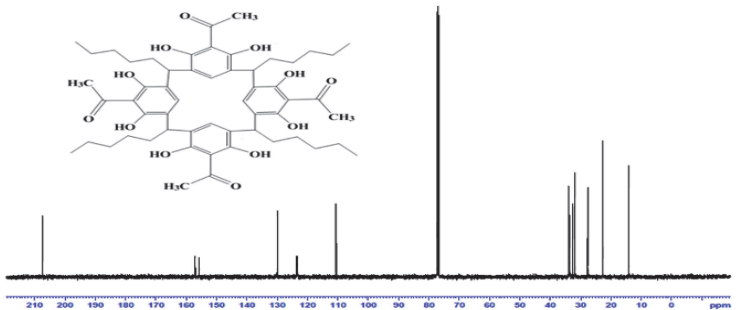

Fig. 10. ${ }^{13} \mathrm{C}-\mathrm{NMR}$ spectrum of compoundld

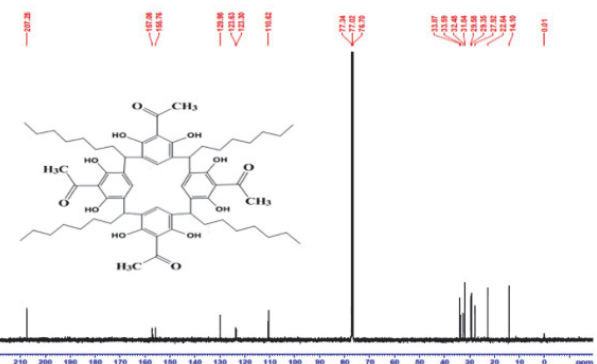

Fig. 12. ${ }^{13} \mathrm{C}-\mathrm{NMR}$ spectrum of compound $1 \mathrm{f}$

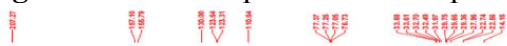

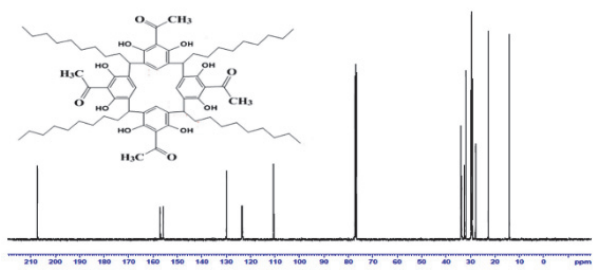

Fig. 14. ${ }^{13} \mathrm{C}-\mathrm{NMR}$ spectrum of compound $1 \mathrm{~h}$

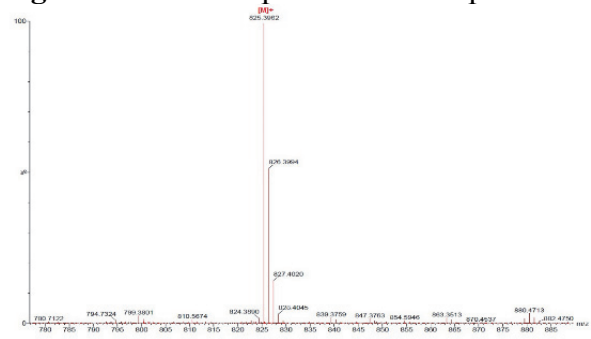

Fig. 16. Mass spectrum of compound $1 \mathrm{~b}$

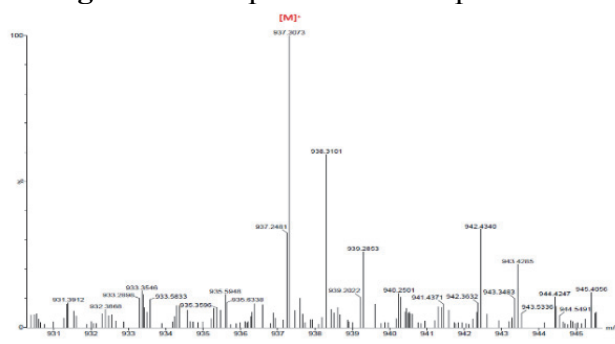

Fig. 18. Mass spectrum of compound 1d

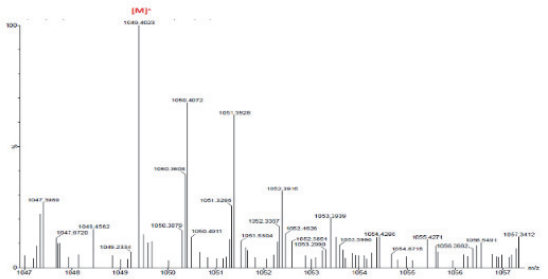

Fig. 20. Mass spectrum of compound if

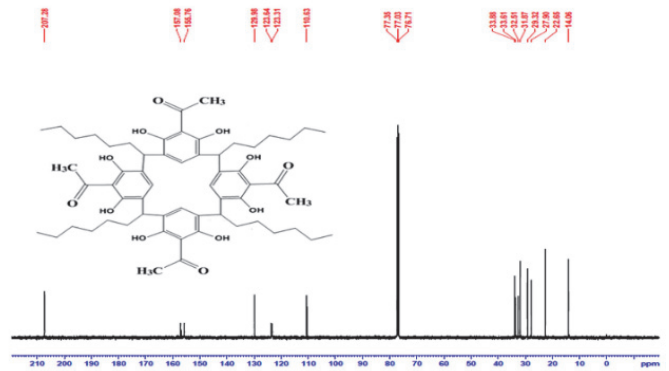

Fig. 11. ${ }^{13} \mathrm{C}$-NMR spectrum of compound $1 \mathrm{e}$

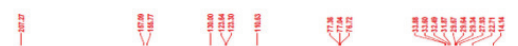

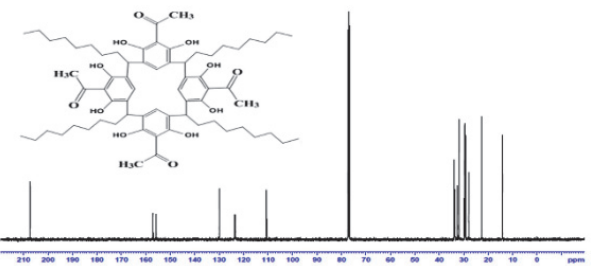

Fig. 13. ${ }^{13} \mathrm{C}-\mathrm{NMR}$ spectrum of compound 1

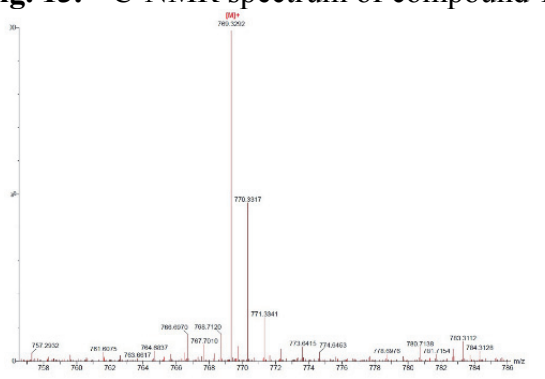

Fig. 15. Mass spectrum of compound 1a

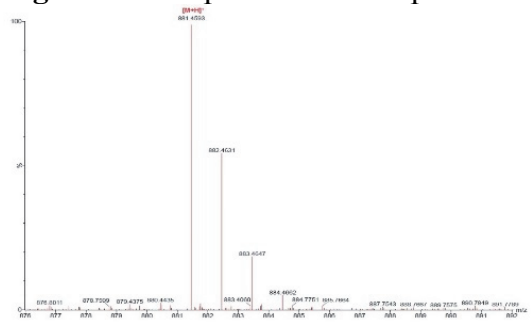

Fig. 17. Mass spectrum of compound 1c

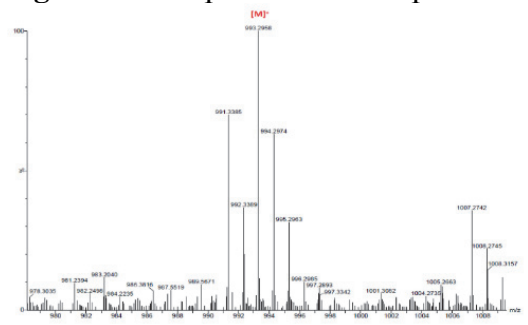

Fig. 19. Mass spectrum of compound 1e

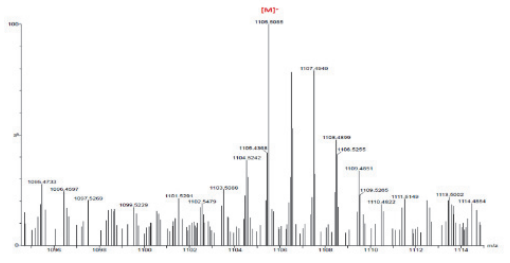

Fig. 21. Mass spectrum of compound $1 \mathrm{~g}$ 


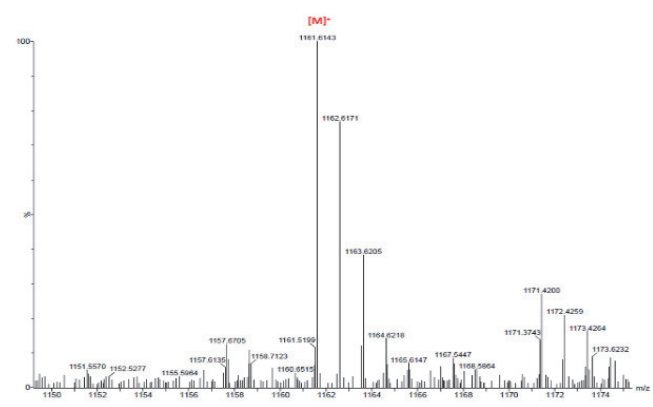

Fig. 22. Mass spectrum of compound $1 \mathrm{~h}$

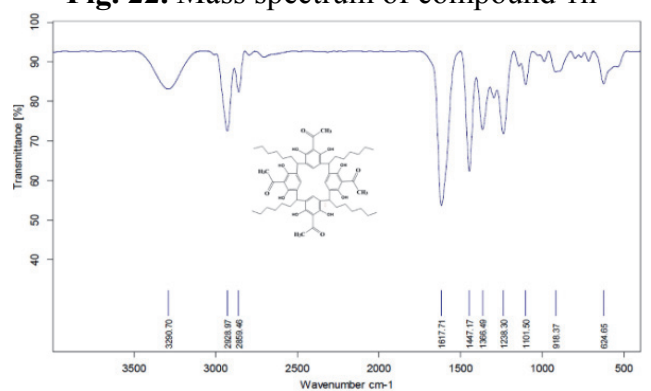

Fig. 24. IR spectrum of compound 1

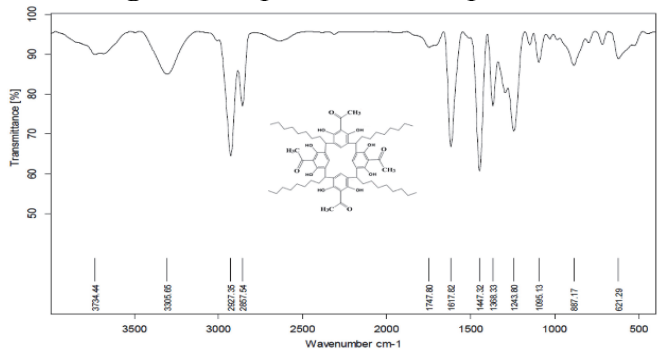

Fig. 26. IR spectrum of compound $1 \mathrm{~g}$

Amino acid detection

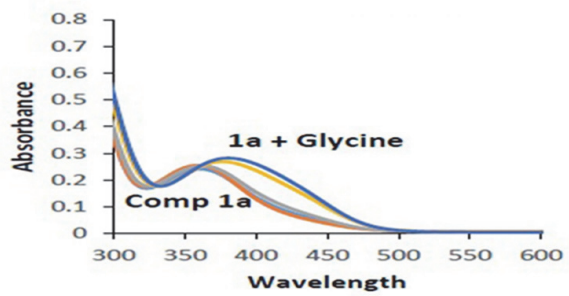

Fig. 28. U.V visible Absorbance spectra of compound 1a with Glycine

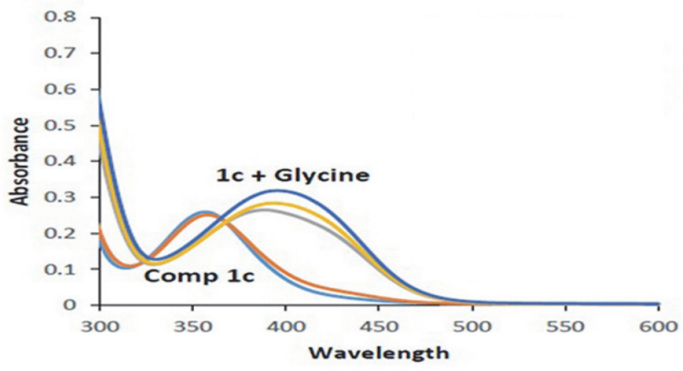

Fig. 30. U.V visible Absorbance spectra of compound 1c with Glycine

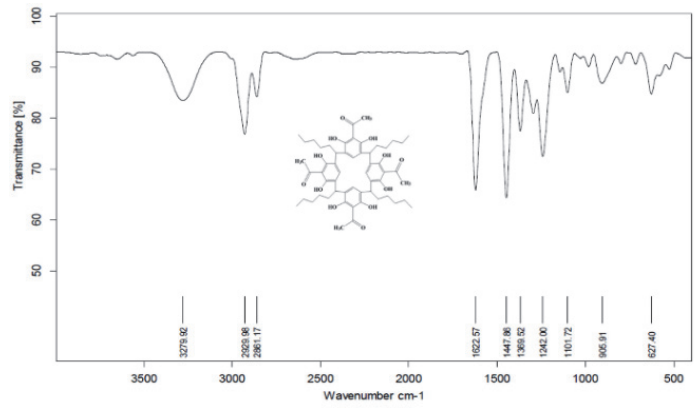

Fig. 23. IR spectrum of compound $1 d$

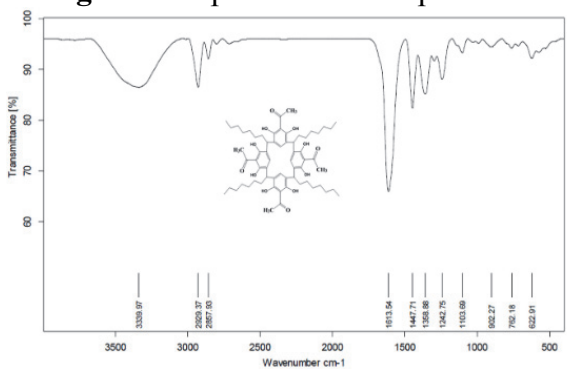

Fig. 25. IR spectrum of compound $1 f$

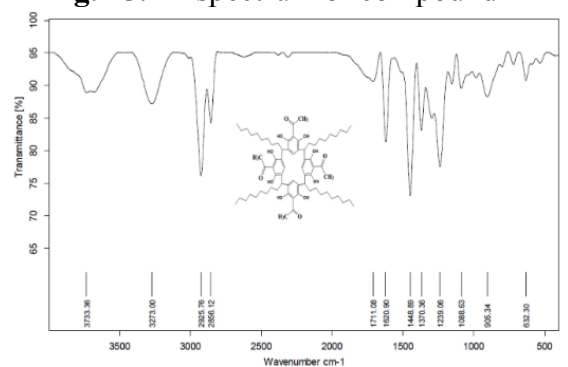

Fig. 27. IR spectrum of compound $1 \mathrm{~h}$

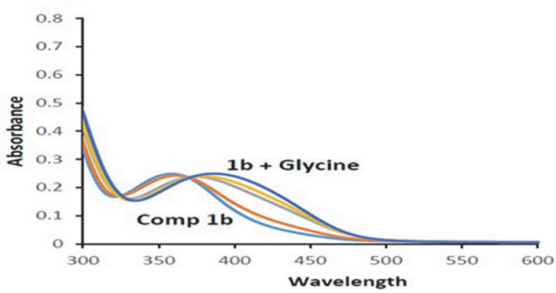

Fig. 29. U.V visible Absorbance spectra of compound 1b with Glycine

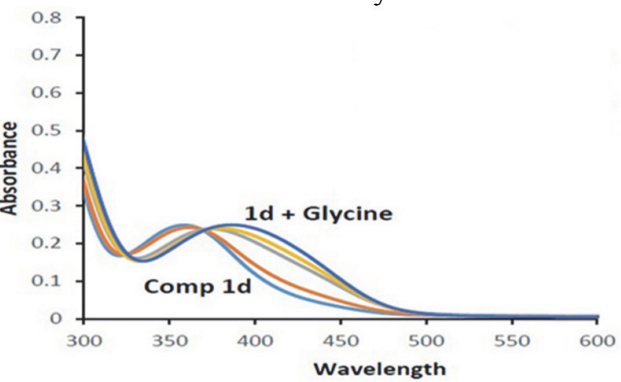

Fig. 31. U.V visible Absorbance spectra of compound 1d with Glycine 


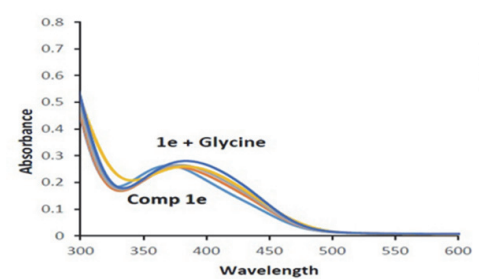

Fig. 32. U.V visible Absorbance spectra of compound 1e with Glycine

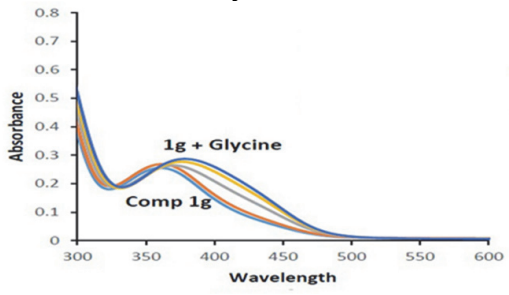

Fig. 34. U.V visible Absorbance spectra of compound $1 \mathrm{~g}$ with Glycine

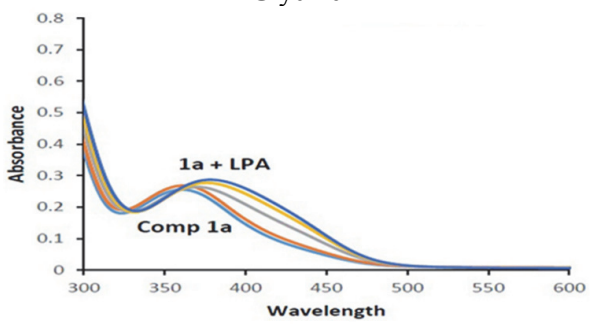

Fig. 36. U.V visible Absorbance spectra of compound 1a with LPA

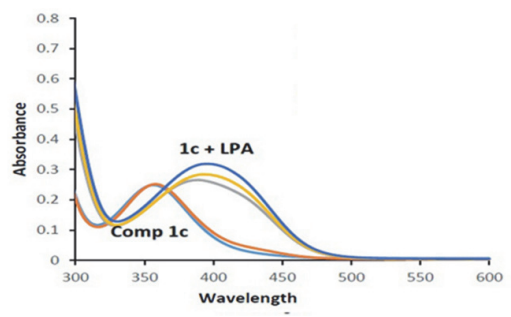

Fig. 38. U.V visible Absorbance spectra of compound 1c with LPA

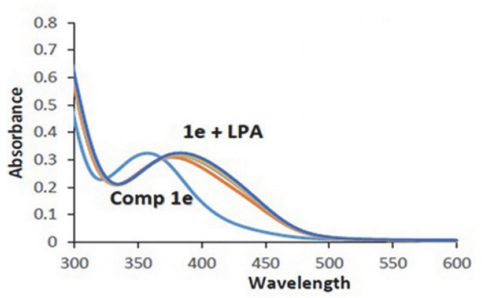

Fig. 40. U.V visible Absorbance spectra of compound 1e with LPA

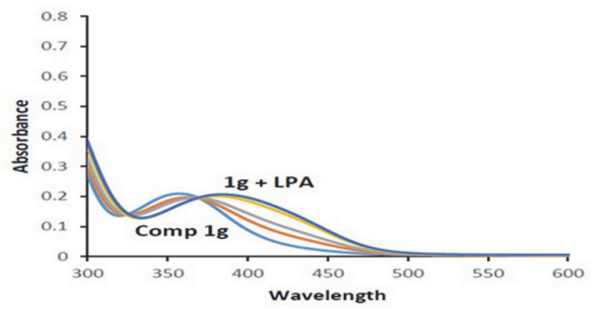

Fig. 42.U.V visible Absorbance spectra of compound $1 \mathrm{~g}$ with LPA

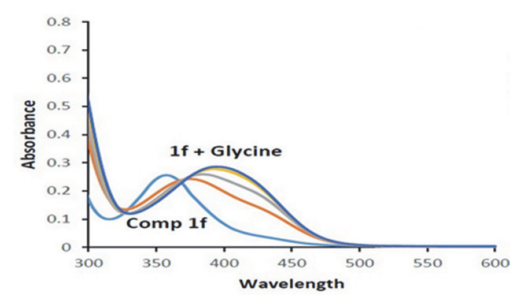

Fig. 33. U.V visible Absorbance spectra of compound $1 \mathrm{f}$ with

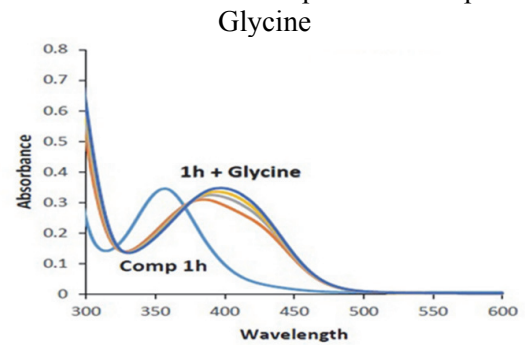

Fig. 35. U.V visible Absorbance spectra of compound $1 \mathrm{~h}$ with Glycine

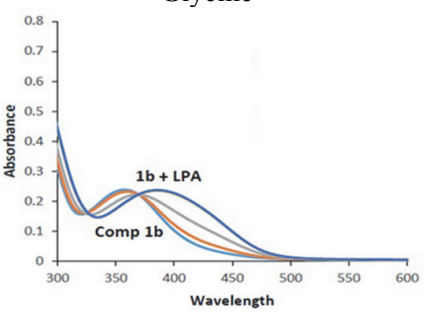

Fig. 37. U.V visible Absorbance spectra of compound $1 b$ with

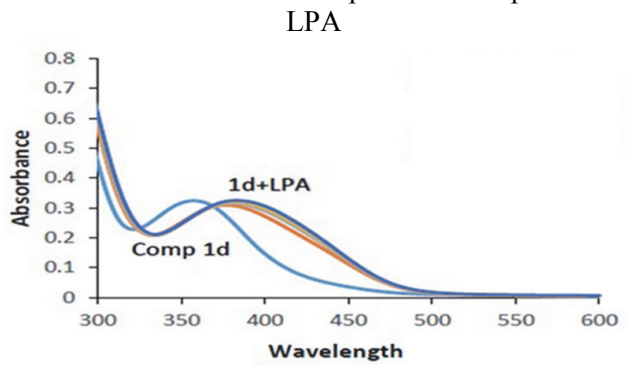

Fig. 39. U.V visible Absorbance spectra of compound 1d with LPA

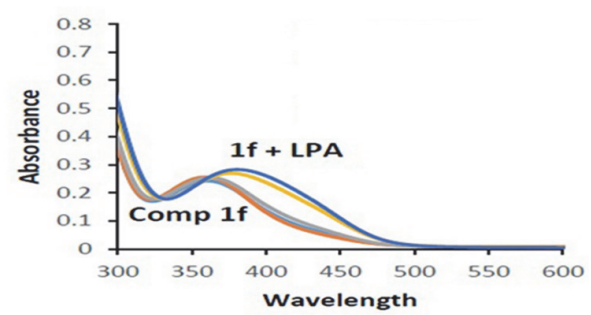

Fig. 41. U.V visible Absorbance spectra of compound $1 \mathrm{f}$ with LPA

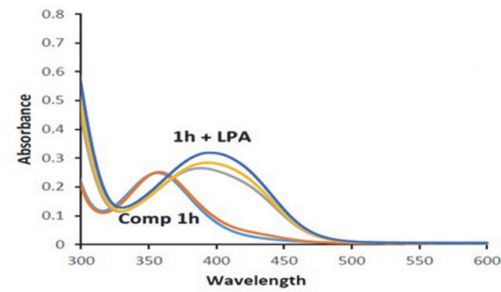

Fig. 43.U.V visible Absorbance spectra of compound 1h with LPA 


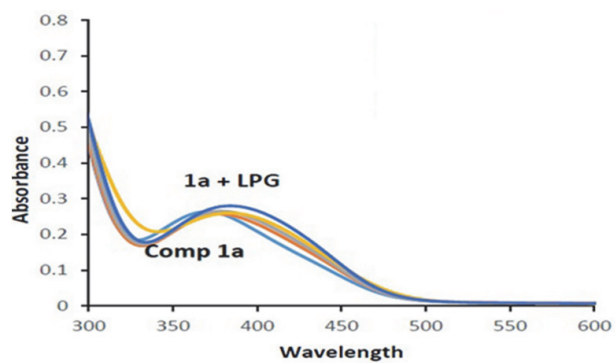

Fig. 44.U.V visible Absorbance spectra of compound 1a

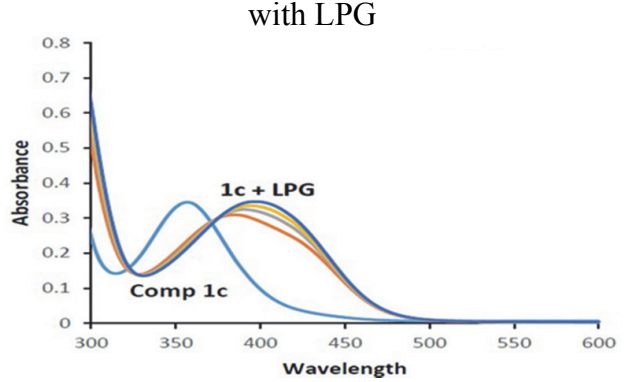

Fig. 46.U.V visible Absorbance spectra of compound 1c with LPG

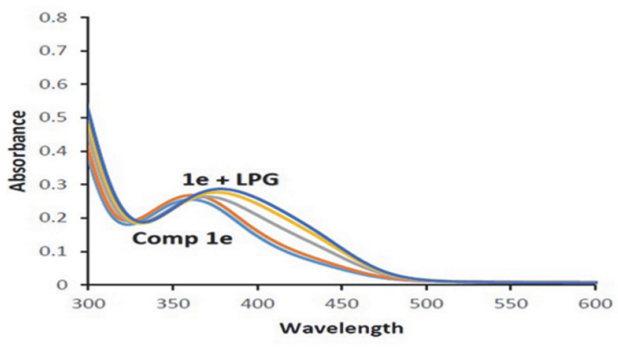

Fig. 48.U.V visible Absorbance spectra of compound 1e with LPG

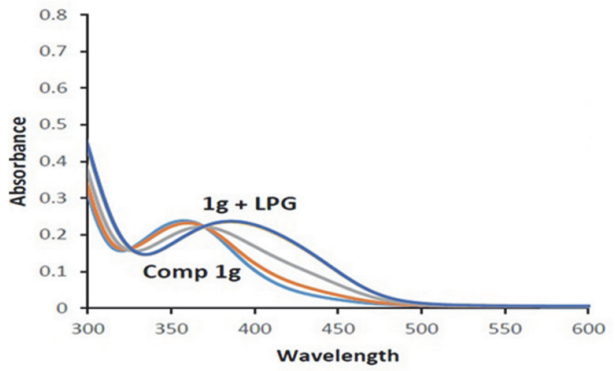

Fig. 50. U.V visible Absorbance spectra of compound $1 \mathrm{~g}$ with LPG

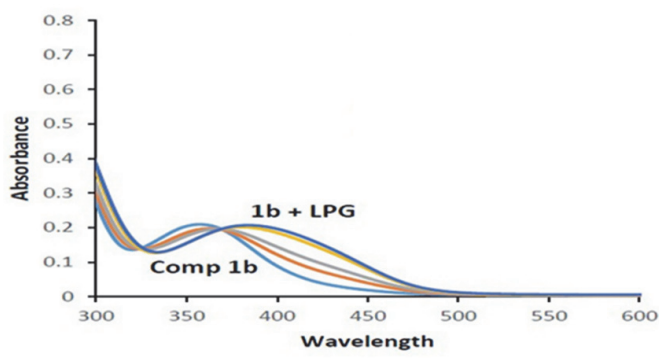

Fig. 45. U.V visible Absorbance spectra of compound $1 \mathrm{~b}$ with LPG

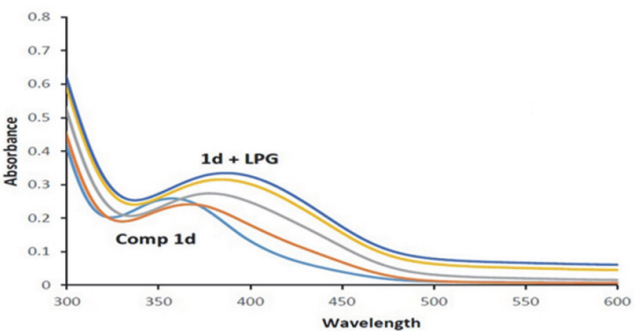

Fig. 47.U.V visible Absorbance spectra of compound 1d

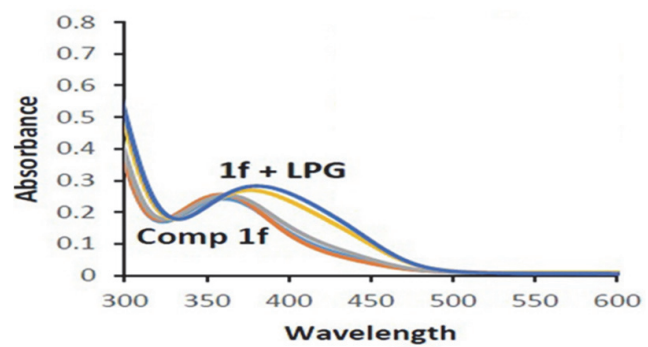

Fig. 49. U.V visible Absorbance spectra of compound if with LPG

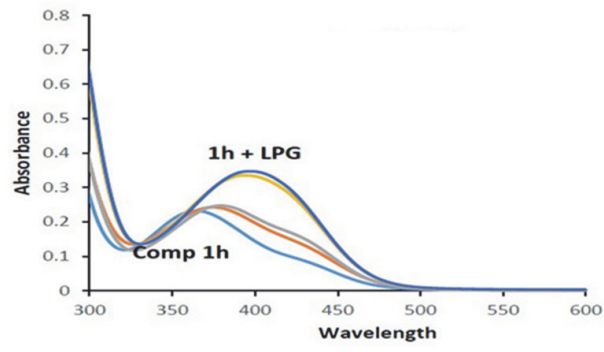

Fig. 51. U.V visible Absorbance spectra of compound $1 \mathrm{~h}$ with LPG

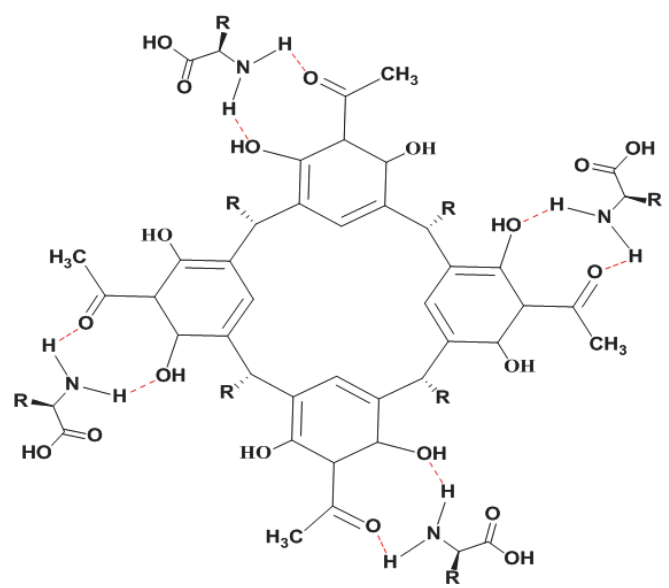

Fig. 52. Proposed structure of the compound after titration with amino acid 


\section{References}

1.Wang, M. (2012) Nitrogen and oxygen bridged calixaromatics: synthesis, structure, functionalization, and molecular recognition. Acc. Chem. Res, Society, 45(2) 182-195.

2.Xu B., Swager T.M. (1993) Rigid Bowlic Liquid Crystals Based on Tungsten-Oxo Calix[4]arenes:Host-Guest Effects and Head-to-Tail Organization. J. Am. Chem. SOC.,115(3) 1159-1160.

3. Steed J.W., Johnson C.P.,Barnes C.L.,Juneja R.K.,Atwood J.L., Reilly S., Hollis R.L., Smith P.H., Clark D. L. (1995)Supramolecular Chemistry of p-Sulfonatocalix[5]arene: A Water-Soluble, BowlShaped Host with a Large Molecular Cavity J. Am. Chem. Soc. 117(46) 11426-11433.

4. Angelova, S., Antonov, L. (2017) Molecular Insight into Inclusion Complex Formation of Curcumin and Calix [4] arene. ChemistrySelect 2(30), 9658-9662.

5. Casnati A., Pochini, A., Ungaro R., Ugozzoli F., Arnaud F., Fanni S., Reinhoudt D. N. (1995) Synthesis, complexation, and membrane transport studies of 1, 3-alternate calix [4] arene-crown-6 conformers: a new class of cesium selective ionophores. J Am Chem Soc. 117(10), 2767-2777.

6. Gutsche C. D., See K. A. (1992) Calixarenes: Synthesis, characterization, and complexation studies of double-cavity calix [4] arenes. J. Org. Chem.57(16), 4527-4539.

7. Gutsche C.D.(2000)Calixarenes for SeparationsACS Symposium Series; J. Am. Chem. Soc. Washington

8. Abosadiya H., Hasbullah S., Mackeen M., Low S., Ibrahim N., Koketsu M., Yamin B. (2013)Synthesis, Characterization, X-ray Structure and Biological Activities of C-5-Bromo-2hydroxyphenylcalix[4]-2-methyl resorcinarene. Molecules, 18(11), 13369-13384.

9. IwanekW., Stefańska K., Szumna,A., Wierzbicki M. (2015) Solvent-free synthesis and structure of 2-naphthol derivatives of resorcinarenes. Tetrahedron, 71(15), 2222-2225.

10. Mobinikhaledi A., Kalhor M., Ghorbani A. R., \& Fathinejad H. (2010) Synthesis of Resorcin [4] arenes Using Microwave Irradiation and Conventional Heating. Asian J. Chem. 22(2), 1103.

11. Konishi H., \& Iwasaki Y. (1995) Base-catalyzed synthesis of a calix [4] resorcinarene: cyclocondensation of 2-butyrylresorcinol with formaldehyde. Synlett, 1995(06), 612-612.

12. Della Sala, P., Gaeta, C., Navarra, W., Talotta, C., De Rosa, M., Brancatelli, G., Neri, P. (2016) Improved Synthesis of Larger Resorcinarenes,J. Org. Chem. 81(13), 5726-5731.

13. Avram L., Cohen Y. (2003) Discrimination of guests encapsulation in large hexameric molecular capsules in solution: pyrogallol [4] arene versus resorcin [4] arene capsules. J Am Chem Soc. 125(52), 16180-16181.

14. Hof F.,Craig S.L.,Nuckolls C.,Rebek J.,(2002)Molecular Encapsulation, Angew.Chem. Int. Ed. 411488-1508.

15. Lu B.B., Jiang W., Yang J., Liu Y.Y., Ma J. F. (2017) Resorcin [4] arene-Based Microporous Metal-Organic Framework as an Efficient Catalyst for $\mathrm{CO} 2$ Cycloaddition with Epoxides and Highly Selective Luminescent Sensing of Cr2O72-. ACS Appl. Mater. Interfaces. 9(45), 3944139449.

16. Chen B., Yang Y., Zapata F., Lin G., Qian G., Lobkovsky E. B. (2007) Luminescent open metal sites within a metal-organic framework for sensing small molecules. Adv. Mater. 19(13), 16931696.

17. Bailey D. M., Hennig A., Uzunova V. D., Nau W. M. (2008) Supramolecular tandem enzyme assays for multiparameter sensor arrays and enantiomeric excess determination of amino acids.Chem Eur. J. 14(20), 6069-6077.

18.Neelakandan P. P., Hariharan,M., Ramaiah, D. (2006) A Supramolecular ON- OFF- ON fluorescence assay for selective recognition of GTP. J. Am. Chem. Soc. 128(35), 11334-11335.

19.Marcotte N., Taglietti A. (2003) Transition-metal-based chemosensing ensembles: ATP sensing in physiological conditions. Supramol Chem, 15(7-8), 617-625.

20. Eliseev A.V., Schneider H. J. (1994) Molecular recognition of nucleotides, nucleosides, and sugars by aminocyclodextrins., J. Am. Chem. Soc.116(14), 6081-6088.

21. Iwanek W., Stefańska K. (2015) A novel, simple and effective synthesis of the hydroxybenzyl 
derivative of resorcinarene and the modification possibilities thereof. Tetrahedron Lett. 56(12), 1496-1500.

22. Hayashida O., Uchiyama M. (2008) Rotaxane-type resorcinarene tetramers as histone-sensing fluorescent receptors. Org. Biomol. Chem. 6(17), 3166-3170.

23. Xu M., Huo F., Yin C. (2017) A supramolecular sensor system to detect amino acids with different carboxyl groups. Sens. Actuator B-Chem. 240, 1245-1250.

24. Tippens A. S., Davis S. V., Hayes J. R., Bryda E. C., Green T. L., Gruetter C. A. (2004) Detection of histidine decarboxylase in rat aorta and cultured rat aortic smooth muscle cells.Inflamm. Res. 53(8), 390-395.

25. Hassen W. M., Martelet C., Davis F., Higson S. P., Abdelghani A., Helali S., \& JaffrezicRenault N. (2007) Calix [4] arene based molecules for amino-acid detection,Sens. Actuator B-Chem. 124(1), 38-45.

26. Glushko V. V., Serkova O. S., Levina I. I., \& Maslennikova V. I.(2016)Tetra-O-acetyl-tetra-Ophosphoryl-tetra-C-naphthyl-resorcin [4] arene. Synthesis and receptor properties toward organic amines. Russ. J. Org. Chem. 52(1), 113-117.

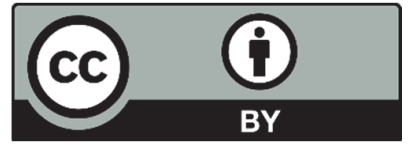

(C) 2019 by the authors; licensee Growing Science, Canada. This is an open access article distributed under the terms and conditions of the Creative Commons Attribution (CC-BY) license (http://creativecommons.org/licenses/by/4.0/). 